\title{
Bioinformatics analysis of FOLR1 expression, functional enrichment, related signaling pathways and relationship with prognosis in ovarian cancer
}

https://doi.org/10.1515/pteridines-2020-0006

received January 2, 2020; accepted February 19, 2020.

\begin{abstract}
Objective To investigate folate-receptor 1 (FOLR1) expression in ovarian cancer and its association
\end{abstract} with patient prognosis.

Methods TCGA and Oncomine databases were used to collect data about FOLR1 mRNA expression in multiple carcinomas. FOLR1 mRNA expression levels in ovarian cancer samples and corresponding adjacent normal ovary tissue were compared. A protein-protein interaction (PPI) network was constructed using the STRING database of FOLR1 and relevant genes. The overall survival (OS) and progression free survival (PFS) rates of ovarian cancer patients in high- and low- FOLR1 expression groups were compared by log-rank test. Sixty-six ovarian epithelial carcinoma samples were included in the study, and tumor specimens of the 66 cases were tested for FOLR1 protein expression by an immunohistochemistry assay. Results FOLR1 mRNA was significantly elevated in ovarian cancer compared to other carcinomas. FOLR1 mRNA expression levels were significantly higher in tumor tissues than in the corresponding normal tissues $(\mathrm{P}<0.05)$ of ovarian cancer patients. The PPI network indicated that the local clustering coefficient was 0.898 , indicating that the PPI network was enriched significantly $(\mathrm{P}<0.05)$. The median PFS values were 22.39 and 19.00 months for lowand high-FOLR1 expression groups, respectively, with significant statistical difference between the two ( $H R=1.26$, 95\%CI:1.09-1.45, $\mathrm{P}<0.05)$. FOLR1 protein expression was correlated with tumor differentiation $(\mathrm{P}<0.05)$ in ovarian

\footnotetext{
*Corresponding author: Pengpeng Qu, Department of Gynecologic Oncology, Tianjin Central Hospital of Gynecology Obstetrics, 300052 PR China, E-mail: qu.pengpeng@hotmail.com

Yan Wang, Xiao Li, Graduate School, Tianjin Medical University, 300070 PR China

Yan Wang, Department of Gynaecology, Jinghai District Hospital of Tianjin 301600 PR China; Department of Gynecologic Oncology, Tianjin Central Hospital of Gynecology Obstetrics, 300052 PR China \# Yan Wang and Xiao Li contribute equally to this work
}

cancer patients. However, its levels were not correlated with patient age, tumor diameter, lymph node metastasis or FIGO stage ( $\mathrm{P}>0.05)$. Conclusion FOLR1 is upregulated in epithelial ovarian cancer, and its expression is correlated with patients' progression free survival, making it a valuable biomarker for prognosis.

Keywords: Epithelial ovarian cancer; bioinformatics analysis; prognosis; immunohistochemistry.

\section{Introduction}

Ovarian cancer is one of the most frequently diagnosed gynecological malignancies, with epithelial ovarian cancer accounting for $80 \%$ to $96 \%$ of all diagnosed ovarian carcinomas [1]. Most patients have developed to a late stage of disease progression by the time of diagnosis; the prognosis is poor, with the 5-year survival rate of $20 \%-30 \%[2,3]$. Therefore, early detection, intervention and accurate evaluation of prognosis are important for ovarian cancer patients. The molecular mechanism of carcinogenesis and development of ovarian cancer is not yet clear [4]. At present, TP53, Rb1, BRCA1 and BRCA2 have been confirmed as cancer-related genes and tumor suppressor genes involved in ovarian cancer, but the survival implications of these genes in ovarian cancer patients are still controversial. About $96 \%$ of epithelial serous ovarian cancers are found to involve TP53 gene mutations [5, 6]. Havrilesky et al. confirmed that TP53 overexpression is not an independent prognostic factor of overall survival (OS) or progression free survival (PFS) in epithelial ovarian cancer patients [7].

Folate receptors are glycosylphosphatidylinositol linker proteins that have high affinity for folate. The Folate receptor encoded by the FOLR gene is a member of the folate receptor family. Members of this gene family bind folic acid and its reduced derivatives and transport 5-methyltetrahydrofolate into cells. Previous studies have 
demonstrated that FOLR1 protein is elevated in the serum of ovarian cancer patients [8]. However, the relationship between FOLR1 expression and patient prognosis is not clear.

\section{Material and methods}

\section{FOLR1 expression examination}

TCGA and Oncomine databases were used to collect data on FOLR1 mRNA expression levels in multiple carcinomas. FOLR1 mRNA expression levels in ovarian cancer samples and corresponding adjacent normal ovary tissues were compared using the online analysis software GEPIA [9]. In parallel, the relationship between relative FOLR1 expression level and clinical stage in ovarian cancer patients was analyzed.

\section{PPI network of FOLR1 construction}

A protein-protein interaction (PPI) network relevant to FOLR1 and associated genes was constructed using the STRING database [10]. The PPI network was constructed using a minimum required interaction score above 0.4 and co-expression, gene function and neighborhood relationship as sources of interaction.

\section{Survival analysis}

According to the median expression of FOLR1 mRNA in ovarian cancer as determined from the TCGA database, patients were divided into two groups: high expression (> median FOLR1 mRNA level) and low expression (< = median FOLR1 mRNA level). The survival difference between high and low expression groups was assessed using the log-rank test.

\section{FOLR1 protein detection by immunohistochemistry}

Sixty-six patients with epithelial ovarian cancer were retrospectively analyzed. Tissue specimens of both tumor tissue and corresponding normal ovary tissue were analyzed for FOLR1 protein expression using an immunohistochemistry assay according to the instructions of the kit.
Table 1: FOLR1 expression score standards.

\begin{tabular}{ll}
\hline Item & Score \\
\hline Stain & \\
Non-staining & 0 \\
Light yellow & 1 \\
Light brown & 2 \\
Brown & 3 \\
Percentage of tumor cells with positive staining & \\
$0 \%$ & 0 \\
$1 \% \sim 25 \%$ & 1 \\
$26 \% \sim 50 \%$ & 2 \\
$51 \% \sim 75 \%$ & 3 \\
$76 \% \sim 100$ & 4 \\
\hline
\end{tabular}

Ethical approval: The research related to human use has been complied with all the relevant national regulations, institutional policies and in accordance the tenets of the Helsinki Declaration, and has been approved by the ethical committee of Jinghai District Hospital of Tianjin. Informed consent: Informed consent has been obtained from all individuals included in this study

\section{FOLR1 protein low and high expression criteria}

Two high-power fields were selected for semi-quantitative scoring by Q-score based on intensity and heterogeneity for specific scoring criteria and scores (Table 1). Each sample's Q-score is the sum of staining intensity and percentage of positive-staining cells. The total scores range from 0 to 7 points. A Q-score $\geq 2$ points was considered to be high expression, and a Q-score $<2$ points was categorized as low expression.

\section{Statistical analysis}

SPSS 22.0 software (SPSS, Inc., Chicago, IL, USA) was used for data analysis. Measurement data was expressed by $\bar{x} \pm s$, and assessed via t test. Counting data was expressed as a raw number and assessed via chi-square test. A two-tailed $\mathrm{p}$-value of $\mathrm{P}<0.05$ was considered to be statistically significant. 

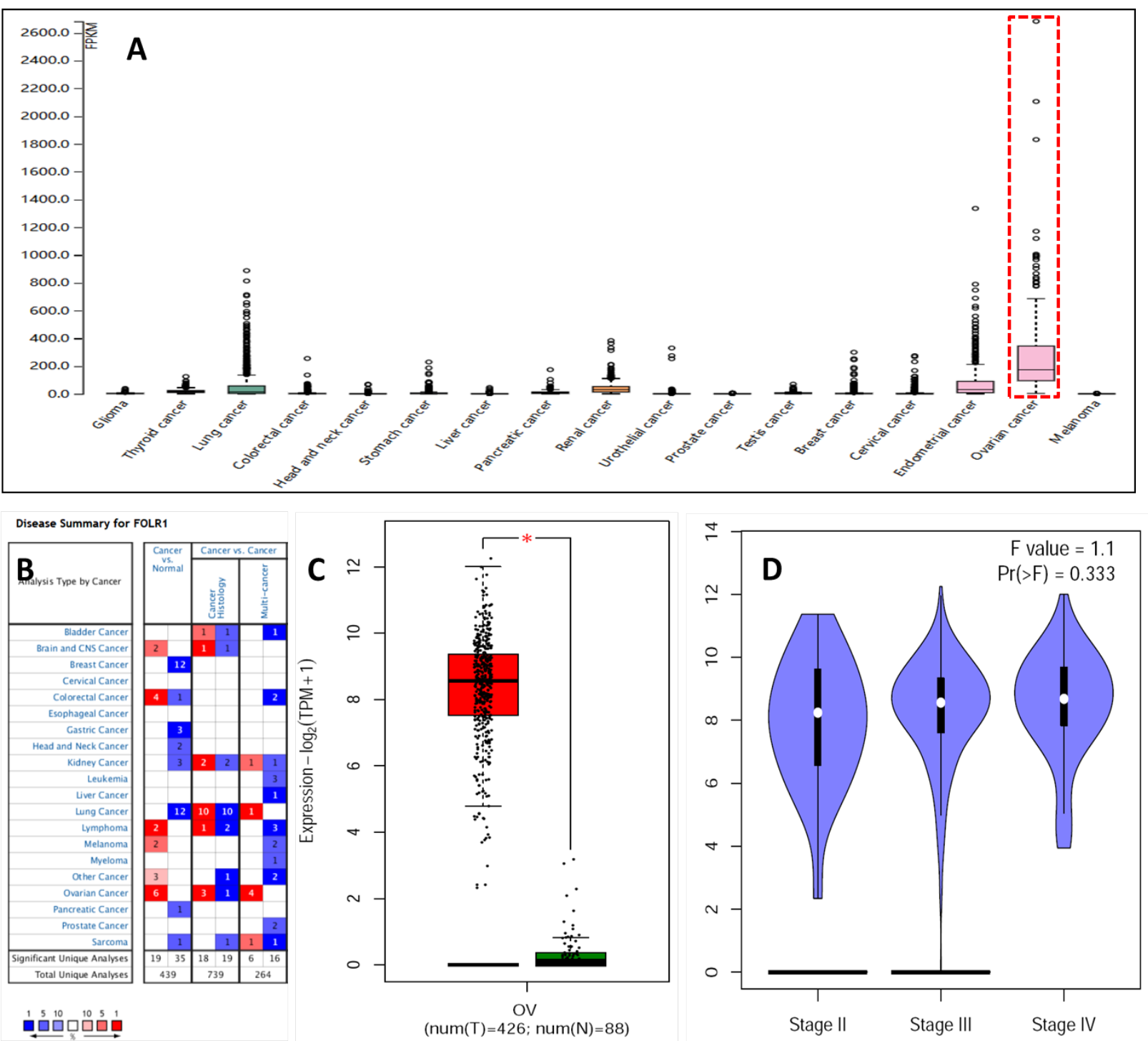

Figure 1: FOLR1 mRNA expression levels in different carcinomas (A: Box plot of FOLR1 mRNA expression levels in different cancers; B: FOLR1 was highly expressed in ovarian cancer tissues as compared to normal ovary tissues according to the Oncomine database; C: FOLR1 mRNA was upregulated in tumor tissues compared to the corresponding normal tissues of ovarian cancer patients according to the TCGA database; D: FOLR1 expression was not different between patients with different stages of ovarian cancer.)

\section{Results}

\section{FOLR1 expression}

FOLR1 mRNA expression was significantly elevated in ovarian cancer compared to other carcinomas (Figure 1A). In the Oncomine database, six studies evaluated FOLR1 expression in ovarian cancer and corresponding normal ovary tissue, and all six studies showed elevated FOLR1 expression level in the cancer tissue as compared to normal tissue (Figure 1B). In the TCGA database, FOLR1 mRNA expression levels were significant higher in cancer tissues than in corresponding normal tissues $(\mathrm{P}<0.05)$, Figure 1C. However, FOLR1 mRNA expression level was not different between different clinical stages of ovarian cancer patients (Figure 1D).

\section{PPI net-work relevant to FOLR1}

Twenty related genes were screened from the protein interaction network. The interaction edge value was 191, with an average interaction index of 13.9. The local clustering coefficient was 0.898 , indicating that the PPI network is significantly enriched $(\mathrm{P}<0.05)$, Figure 2. Of 


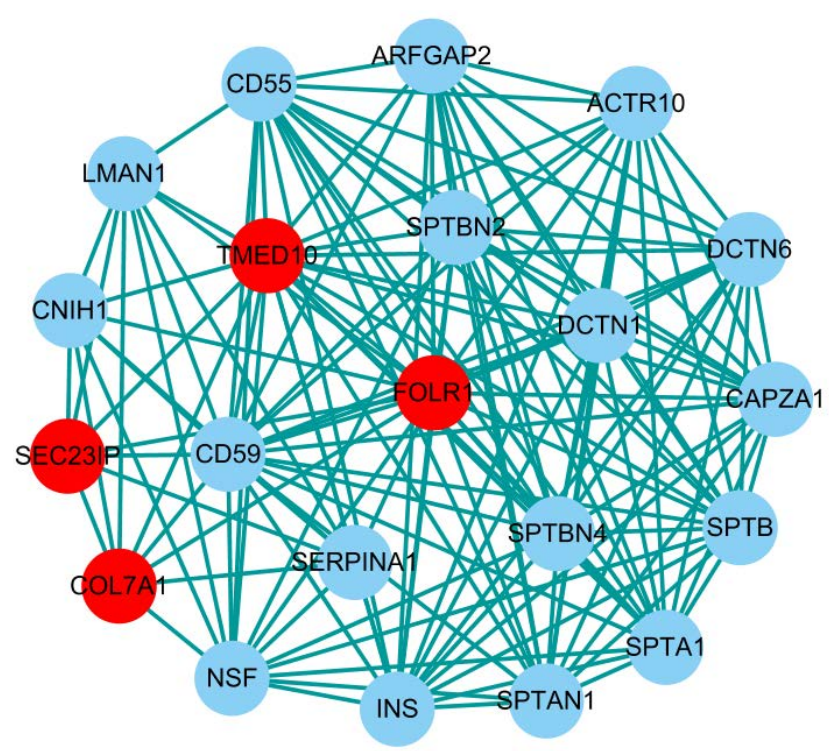

Figure2: PPI network relevant to FOLR1 and correlated genes (Red nodes indicate hub genes of the network).

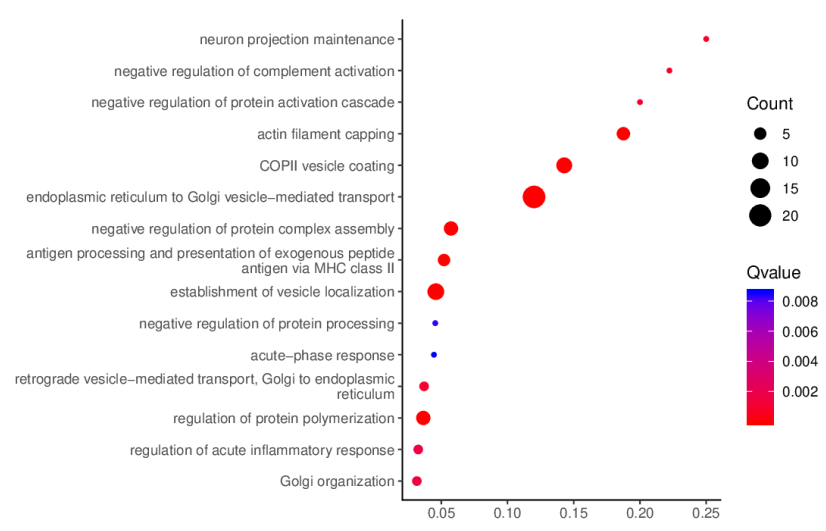

Figure 3: Bubble plot of biological process enrichment for FOLR1 and relevant genes.

the 21 genes included in the PPI network, four hub genes (FOLR1, TMED10, SEC23IP and COL7A1) were identified.

\section{GO and enrichment}

GO enrichment of FOLR1 and relevant genes is shown in Table 2. With regards to biological processes, FOLR1 and correlated genes were enriched in neuron projection maintenance, establishment of vesicle localization, negative regulation of protein activation cascade, etc. (Figure 3). With regards to cellular components, FOLR1 and related genes were enriched in spectrin, dynactin complex, spectrin-associated cytoskeleton, etc. (Figure 4). With regards to molecular function, FOLR1 and

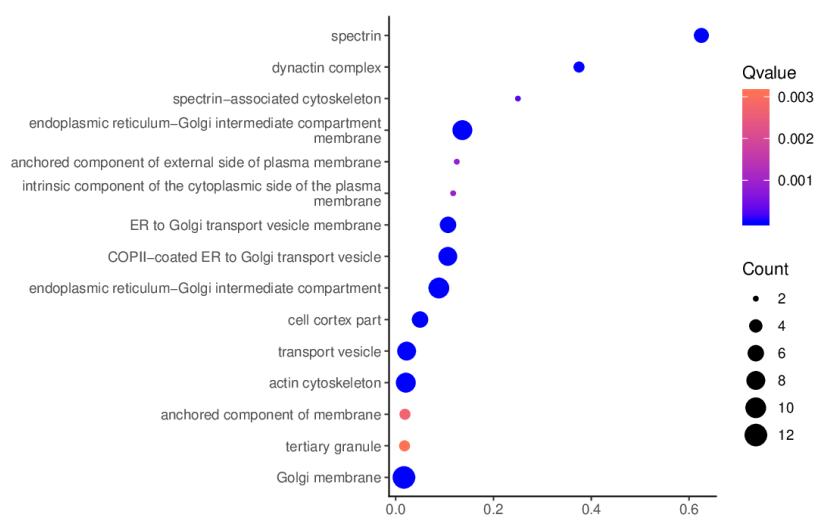

Figure 4: Bubble plot of cellular component enrichment for FOLR1 and relevant genes.

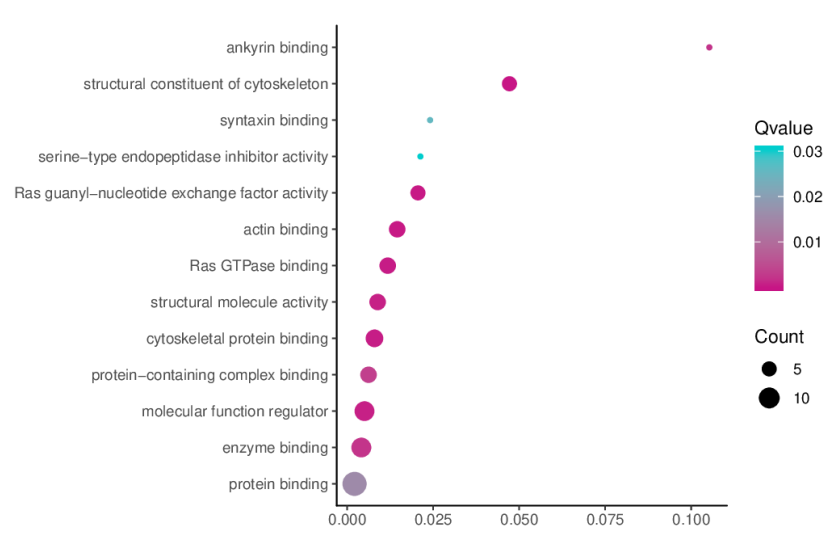

Figure 5: Bubble plot of molecular function enrichment for FOLR1 and relevant genes.

correlated genes were enriched in structural constituents of cytoskeleton actin binding and Ras guanyl-nucleotide exchange factor activity (Figure 5).

\section{KEGG pathway enrichment}

FOLR1 and relevant genes were mainly enriched in the vasopressin-regulated water reabsorption, complement and coagulation cascades, the hematopoietic cell lineage and the endocytosis pathway, Table 3. Figure 6.

\section{Survival analysis}

The median progression free survival (PFS) values were 22.39 and 19.00 months for FOLR1 low and high expression groups, respectively, with a statistically significant difference between the two (HR=1.26, 95\%CI:1.09-1.45, $\mathrm{P}<0.05$ ), Figure 7. However, the overall survival (OS) 
Table 2: GO enrichment of FOLR1 and relevant genes in the aspects of $\mathrm{BP}, \mathrm{CC}$ and MF.

\begin{tabular}{|c|c|c|c|}
\hline Description & Count & P value & Gene Ratio \\
\hline \multicolumn{4}{|l|}{ BP } \\
\hline neuron projection maintenance & 2 & 0.00061 & 0.25 \\
\hline negative regulation of complement activation & 2 & 0.00073 & 0.222222 \\
\hline negative regulation of protein activation cascade & 2 & 0.00086 & 0.2 \\
\hline actin filament capping & 6 & $9.49 \mathrm{E}-11$ & 0.1875 \\
\hline COPII vesicle coating & 9 & $3.00 \mathrm{E}-15$ & 0.142857 \\
\hline endoplasmic reticulum to Golgi vesicle-mediated transport & 21 & $3.42 \mathrm{E}-40$ & 0.12 \\
\hline negative regulation of protein complex assembly & 7 & $1.84 \mathrm{E}-09$ & 0.057377 \\
\hline antigen processing and presentation of exogenous peptide antigen via MHC class II & 5 & $1.48 \mathrm{E}-06$ & 0.052083 \\
\hline establishment of vesicle localization & 10 & $7.49 \mathrm{E}-13$ & 0.045872 \\
\hline negative regulation of protein processing & 2 & 0.0084 & 0.045455 \\
\hline acute-phase response & 2 & 0.0086 & 0.044444 \\
\hline retrograde vesicle-mediated transport, Golgi to endoplasmic reticulum & 3 & 0.0011 & 0.037037 \\
\hline regulation of protein polymerization & 7 & $3.18 \mathrm{E}-08$ & 0.036458 \\
\hline regulation of acute inflammatory response & 3 & 0.0015 & 0.032609 \\
\hline Golgi organization & 3 & 0.0015 & 0.031579 \\
\hline \multicolumn{4}{|l|}{ CC } \\
\hline spectrin & 5 & $6.35 \mathrm{E}-11$ & 0.625 \\
\hline dynactin complex & 3 & $1.57 \mathrm{E}-06$ & 0.375 \\
\hline spectrin-associated cytoskeleton & 2 & 0.00028 & 0.25 \\
\hline endoplasmic reticulum-Golgi intermediate compartment membrane & 9 & $5.11 \mathrm{E}-15$ & 0.136364 \\
\hline anchored component of external side of plasma membrane & 2 & 0.00084 & 0.125 \\
\hline intrinsic component of the cytoplasmic side of the plasma membrane & 2 & 0.00092 & 0.117647 \\
\hline ER to Golgi transport vesicle membrane & 6 & $1.07 \mathrm{E}-09$ & 0.107143 \\
\hline COPII-coated ER to Golgi transport vesicle & 8 & $1.12 \mathrm{E}-12$ & 0.106667 \\
\hline endoplasmic reticulum-Golgi intermediate compartment & 10 & $5.11 \mathrm{E}-15$ & 0.088496 \\
\hline cell cortex part & 6 & 4.92E-08 & 0.05 \\
\hline transport vesicle & 8 & $3.63 \mathrm{E}-08$ & 0.022472 \\
\hline actin cytoskeleton & 9 & $6.43 \mathrm{E}-09$ & 0.020833 \\
\hline anchored component of membrane & 3 & 0.0027 & 0.019231 \\
\hline tertiary granule & 3 & 0.0031 & 0.018293 \\
\hline Golgi membrane & 12 & $6.35 \mathrm{E}-11$ & 0.016854 \\
\hline \multicolumn{4}{|l|}{ MF } \\
\hline structural constituent of cytoskeleton & 5 & $1.05 \mathrm{E}-05$ & 0.04717 \\
\hline actin binding & 6 & 0.0002 & 0.014528 \\
\hline Ras guanyl-nucleotide exchange factor activity & 5 & 0.0002 & 0.020576 \\
\hline Ras GTPase binding & 6 & 0.00033 & 0.011765 \\
\hline
\end{tabular}


Continued Table 2: GO enrichment of FOLR1 and relevant genes in the aspects of $\mathrm{BP}, \mathrm{CC}$ and MF.

\begin{tabular}{|c|c|c|c|}
\hline Description & Count & Pvalue & Gene Ratio \\
\hline cytoskeletal protein binding & 7 & 0.00038 & 0.007937 \\
\hline molecular function regulator & 9 & 0.00056 & 0.00502 \\
\hline structural molecule activity & 6 & 0.00064 & 0.008837 \\
\hline enzyme binding & 9 & 0.0021 & 0.004096 \\
\hline ankyrin binding & 2 & 0.0021 & 0.105263 \\
\hline protein-containing complex binding & 6 & 0.0034 & 0.006198 \\
\hline protein binding & 14 & 0.0157 & 0.00212 \\
\hline syntaxin binding & 2 & 0.0257 & 0.024096 \\
\hline serine-type endopeptidase inhibitor activity & 2 & 0.0305 & 0.021277 \\
\hline
\end{tabular}

Table 3: KEGG pathway enrichment of FOLR1 and relevant genes in the aspects of $\mathrm{BP}, \mathrm{CC}$ and MF.

\begin{tabular}{lllll}
\hline Description & Count & Background gene & P value & Gene Ratio \\
\hline Vasopressin-regulated water reabsorption & 3 & 44 & 0.00067 & 0.068182 \\
Complement and coagulation cascades & 3 & 78 & 0.0017 & 0.038462 \\
Hematopoietic cell lineage & 2 & 94 & 0.047 & 0.021277 \\
Endocytosis & 3 & 242 & 0.029 & 0.012397 \\
\hline
\end{tabular}

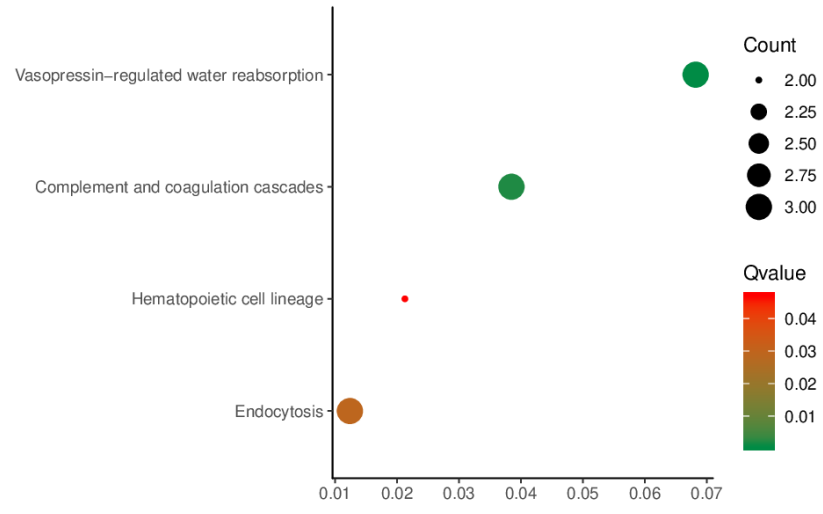

Figure 6: Bubble plot of KEGG pathway enrichment for FOLR1 and relevant genes.

values were not significantly different between FOLR1-low (median 42.6 months) and -high (median 48.37 months) groups (HR=0.89, 95\%CI:0378-1.02, P>0.05), Figure 7 .

\section{Immunohistochemistry assay}

Immunohistochemical staining showed that FOLR1 protein is mainly expressed in the nucleus, with positive expression apparent as yellow brown granules evenly distributed throughout the nucleus. Of the included 66 cases, 29 (43.9\%) were found positive for expressed FOLR1 protein, Figure 8.

\section{FOLR1 protein expression and patients' clinical characteristics}

FOLR1 protein expression was correlated with tumor differentiation $(\mathrm{P}<0.05)$ of ovarian cancer patients. However, expression was not correlated with patient age, tumor diameter, lymph node metastasis or FIGO stage ( $P>0.05)$, Table 4.

\section{Discussion}

Ovarian cancer is one of the most frequently diagnosed gynecological malignant tumors and has a high mortality rate. Due to the lack of obvious symptoms, early diagnosis is difficult, and overall disease prognosis is poor. Notably, general prognosis is quite different for different patients, reflecting significant biological heterogeneity across 

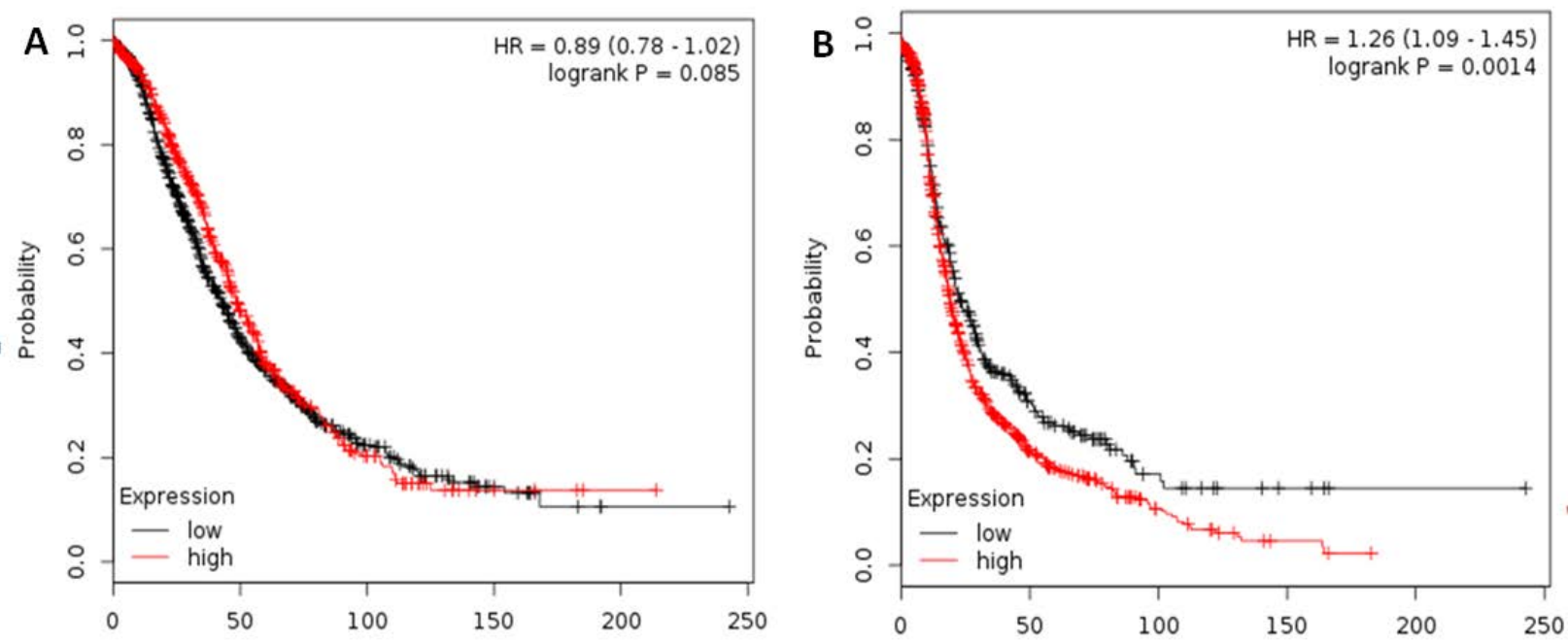

Figure 7: Survival curves of ovarian cancer patients in high and low FOLR1 mRNA expression groups (A: overall survival; B: progression-free survival).
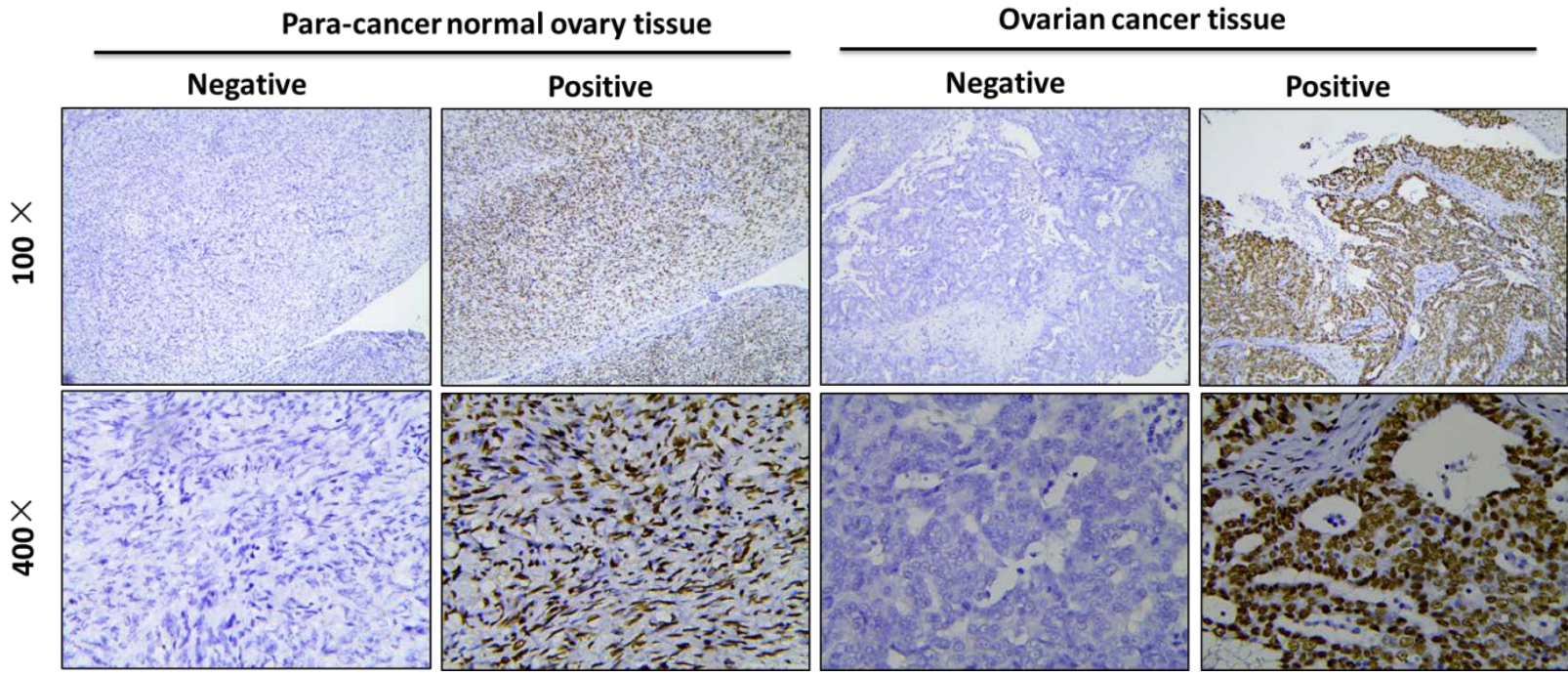

Figure 8: Immunohistochemistry assay used to assess FOLR1 expression in ovarian cancer tissue and normal ovarian tissue.

ovarian cancer cases. More and more evidence has confirmed that the management of ovarian cancer should be personalized, taking into account the performance status of the patient; this is particularly highlighted by cases of elderly women, who show quite different disease trajectories that younger patients [11, 12]. Studies have also demonstrated that some genes' expression profiles are associated with prognosis of ovarian cancer patients; these genes include tp53, miR-34a [13], etc. Recent publications have indicated that FOLR1 is upregulated in multiple cancers and may participate in carcinogenesis [14-17]. Studies have confirmed that FOLR1 is elevated in the serum of ovarian cancer patients as compared to that of healthy subjects or those with benign ovarian tumors [8]. However, it remains unclear whether FOLR1 is upregulated in the tumor tissue of ovarian cancer patients, as well as whether its expression level is correlated with the patient's prognosis. Therefore, we carried out this bioinformatics study to evaluate FOLR1 expression, functional enrichment, related signaling pathways and relationship with patients' prognoses in ovarian cancer, then further validated these results through an immunohistochemistry assay. 
Table 4: FOLR1 protein expression and clinical pathological features of ovarian cancer patients.

\begin{tabular}{|c|c|c|c|c|c|}
\hline \multirow[t]{2}{*}{ Features } & \multirow[t]{2}{*}{$n=66$} & \multicolumn{2}{|c|}{ FOLR1 expression } & \multirow[t]{2}{*}{$x^{2}$} & \multirow[t]{2}{*}{$P$} \\
\hline & & $\begin{array}{l}\text { Positive } \\
(n=29)\end{array}$ & $\begin{array}{l}\text { Negative } \\
(\mathrm{n}=37)\end{array}$ & & \\
\hline Age (year) & & & & 1.18 & 0.28 \\
\hline$<=60$ & 36 & 18 & 18 & & \\
\hline$>60$ & 30 & 11 & 19 & & \\
\hline $\begin{array}{l}\text { Tumor } \\
\text { diameter }(\mathrm{cm})\end{array}$ & & & & 0.14 & 0.71 \\
\hline$<=7$ & 29 & 12 & 17 & & \\
\hline$>7$ & 37 & 17 & 20 & & \\
\hline Differentiation & & & & 5.73 & 0.02 \\
\hline $\begin{array}{l}\text { Well/ } \\
\text { moderate }\end{array}$ & 36 & 9 & 17 & & \\
\hline Poor & 30 & 20 & 10 & & \\
\hline $\begin{array}{l}\text { Lymph node } \\
\text { metastasis }\end{array}$ & & & & 0.73 & 0.39 \\
\hline Negative & 28 & 14 & 14 & & \\
\hline Positive & 38 & 15 & 23 & & \\
\hline FIGO stage & & & & 2.09 & 0.15 \\
\hline $\mathrm{I} / \mathrm{II}$ & 27 & 9 & 18 & & \\
\hline III/IV & 39 & 20 & 19 & & \\
\hline
\end{tabular}

In the present work, we found that FOLR1 mRNA levels are significantly elevated in ovarian cancer compared to other carcinomas. FOLR1 mRNA expression levels were significantly higher in tumor tissues than in corresponding normal tissues $(\mathrm{P}<0.05)$ of ovarian cancer patients. This result demonstrates that increased FOLR1 mRNA expression is specific to ovarian cancer and may therefore participate in ovarian cancer development. Survival analysis showed that median progression free survival (PFS) was significant longer in the FOLR1 lowexpression group compared to the high-expression group. FOLR1 mRNA can be used as a biomarker for poor PFS in ovarian cancer patients.

Folate is involved in intracellular metabolism, as well as DNA synthesis and repair, and it is an essential factor for both normal and tumor cells [18-20]. FOLR1, a membrane protein with a molecular weight of 38-40 KD, easily binds oxidized folate, which is not expressed in normal cells, but is expressed by a large number of nonmucinous epithelial tumor cells (such as epithelial ovarian cancer cells). However, the mechanism responsible for this high expression of FOLR1 in tumor cells is still unclear
[21-23]. It is generally believed that protein is either used to regulate the amount of folate taken up from serum or as a participant in signal regulation during carcinoma development. Although the role of FOLR1 in tumor cells is still unclear, it has been reported to be highly expressed in ovarian cancer [24, 25], cervical cancer [14], liver cancer [26] and breast cancer [22, 27]. The soluble FOLR1 expressed on the cell surface can be released by the binding of a membrane protease with glycosylphosphatidylinositol specific phospholipase $\mathrm{C}$ and $\mathrm{D}$.

A possible mechanism underlying the high expression of FOLR1 in ovarian cancer is the following [28]: (1) FOLR1 can promote tumor growth by positively regulating folate uptake from plasma, which is conducive to rapid cell growth and division; (2) In the early stages of carcinogenesis, FOLR1 up-regulation increases absorption of folic acid to stimulate repair of damaged DNA; (3) When damaged DNA cannot be repaired, sustained expression of FOLR1 is induced, ultimately generating a cellular environment that is conducive to tumor progression. In order to grow rapidly, the tumor will increase its demand for folate.

Another factor affecting the prognosis of ovarian cancer patients is recurrence after surgery or firstline chemotherapy. Recurrence of the disease usually responds poorly to first-, and sometimes even second-, line chemotherapies. In this regard, it is possible that this inherent ovarian cancer resistance may be due to reduced immunosurveillance and drug-resistant cells $[29,30]$.

Our results indicate that FOLR1 is upregulated in epithelial ovarian cancer, and expression levels are correlated with patients' progression free survival. Therefore, FOLR1 may be a potential biomarker for predicting the prognosis of ovarian cancer patients. Elevated FOLR1 in ovarian cancer patients would be a risk factor for lowered progression free survival. However, there are two important limitations to the present work: 1) The molecular biological function of FOLR1 in ovarian cancer development and its specific contribution to poor prognosis are not clear and need further investigation; 2) All results and conclusions were based on bioinformatic analysis and will require further validation by cellular experiments.

Conflict of interest: Authors state no conflict of interest 


\section{References}

1. Siegel RL, Miller KD, Jemal A. Cancer statistics, 2019. CA Cancer J Clin. 2019 Jan;69(1):7-34.

2. Torre LA, Trabert B, DeSantis CE, Miller KD, Samimi G, Runowicz CD, et al. Ovarian cancer statistics, 2018. CA Cancer J Clin. 2018 Jul;68(4):284-96.

3. KRAUL L. Krebsarzt. 1952;7:289-91.

4. Liu J, Meng H, Li S, Shen Y, Wang H, Shan W, et al. Identification of Potential Biomarkers in Association With Progression and Prognosis in Epithelial Ovarian Cancer by Integrated Bioinformatics Analysis. Front Genet. 2019 Oct;10:1031.

5. Cipriano NM Jr, de Brito AM, de Oliveira ES, de Faria FC, Lemos $\mathrm{S}$, Rodrigues AN, et al. Mutation screening of TP53, CHEK2 and BRCA genes in patients at high risk for hereditary breast and ovarian cancer (HBOC) in Brazil. Breast Cancer. 2019 May;26(3):397-405.

6. Hu J, Liu Z, Wang X. Does TP53 mutation promote ovarian cancer metastasis to omentum by regulating lipid metabolism? Med Hypotheses. 2013 Oct;81(4):515-20.

7. Havrilesky L, Darcy M, Hamdan H, Priore RL, Leon J, Bell J, et al.; Gynecologic Oncology Group Study. Prognostic significance of p53 mutation and p53 overexpression in advanced epithelial ovarian cancer: a Gynecologic Oncology Group Study. J Clin Oncol. 2003 Oct;21(20):3814-25.

8. Leung F, Dimitromanolakis A, Kobayashi H, Diamandis EP, Kulasingam V. Folate-receptor 1 (FOLR1) protein is elevated in the serum of ovarian cancer patients. Clin Biochem. 2013 Oct;46(15):1462-8.

9. Tang Z, Li C, Kang B, Gao G, Li C, Zhang Z. GEPIA: a web server for cancer and normal gene expression profiling and interactive analyses [J]. Nucleic Acids Res. 2017 Jul;45 W1:W98-102.

10. Szklarczyk D, Gable AL, Lyon D, Junge A, Wyder S, Huerta-Cepas J, et al. STRING v11: protein-protein association networks with increased coverage, supporting functional discovery in genome-wide experimental datasets [J]. Nucleic Acids Res. 2019 Jan;47 D1:D607-13.

11. Vitale SG, Capriglione S, Zito G, Lopez S, Gulino FA, Di Guardo $\mathrm{F}$, et al. Management of endometrial, ovarian and cervical cancer in the elderly: current approach to a challenging condition. Arch Gynecol Obstet. 2019 Feb;299(2):299-315.

12. Schuurman MS, Kruitwagen RF, Portielje JE, Roes EM, Lemmens VE, van der Aa MA. Treatment and outcome of elderly patients with advanced stage ovarian cancer: A nationwide analysis. Gynecol Oncol. 2018 May;149(2):270-4.

13. Schmid G, Notaro S, Reimer D, Abdel-Azim S, Duggan-Peer M, Holly J, et al. Expression and promotor hypermethylation of miR-34a in the various histological subtypes of ovarian cancer. BMC Cancer. 2016 Feb;16(1):102.

14. Liu C, Ding L, Bai L, Chen X, Kang H, Hou L, et al. Folate receptor alpha is associated with cervical carcinogenesis and regulates cervical cancer cells growth by activating ERK1/2/c-Fos/c-Jun. Biochem Biophys Res Commun. 2017 Sep;491(4):1083-91.

15. Boogerd LS, Hoogstins CE, Gaarenstroom KN, de Kroon CD, Beltman JJ, Bosse T, et al. Folate receptor- $\alpha$ targeted nearinfrared fluorescence imaging in high-risk endometrial cancer patients: a tissue microarray and clinical feasibility study. Oncotarget. 2017 Dec;9(1):791-801.
16. Shen J, Hu Y, Putt KS, Singhal S, Han H, Visscher DW, et al. Assessment of folate receptor alpha and beta expression in selection of lung and pancreatic cancer patients for receptor targeted therapies. Oncotarget. 2017 Dec;9(4):4485-95.

17. Omote S, Takata K, Tanaka T, Miyata-Takata T, Ayada Y, Noujima-Harada M, et al. Overexpression of folate receptor alpha is an independent prognostic factor for outcomes of pancreatic cancer patients. Med Mol Morphol. 2018 Dec;51(4):237-43.

18. Pérez-Ramírez C, Cañadas-Garre M, Alnatsha A, Villar E, Delgado JR, Calleja-Hernández MÁ, et al. Impact of DNA repair, folate and glutathione gene polymorphisms on risk of non small cell lung cancer. Pathol Res Pract. 2018 Jan;214(1):44-52.

19. Zhao H, Zeng X. Clinical influence of maternal serum homocysteine, folate and vitamin B12 in the development of preeclampsia. Pteridines. 2019;30(1):48-53.

20. Wei Q, Shen H, Wang LE, Duphorne CM, Pillow PC, Guo Z, et al. Association between low dietary folate intake and suboptimal cellular DNA repair capacity. Cancer Epidemiol Biomarkers Prev. 2003 Oct;12(10):963-9.

21. Cheung A, Opzoomer J, Ilieva KM, Gazinska P, Hoffmann RM, Mirza H, et al. Anti-Folate Receptor Alpha-Directed Antibody Therapies Restrict the Growth of Triple-negative Breast Cancer. Clin Cancer Res. 2018 Oct;24(20):5098-111.

22. Ginter PS, McIntire PJ, Cui X, Irshaid L, Liu Y, Chen Z, et al. Folate Receptor Alpha Expression Is Associated With Increased Risk of Recurrence in Triple-negative Breast Cancer. Clin Breast Cancer. 2017 Nov;17(7):544-9.

23. Cheung A, Bax HJ, Josephs DH, Ilieva KM, Pellizzari G, Opzoomer J, et al. Targeting folate receptor alpha for cancer treatment. Oncotarget. 2016 Aug;7(32):52553-74.

24. Farran B, Albayrak S, Abrams J, Tainsky MA, Levin NK, Morris R, et al. Serum folate receptor $\alpha(\mathrm{sFR})$ in ovarian cancer diagnosis and surveillance. Cancer Med. 2019 Mar;8(3):920-7.

25. Kurosaki A, Hasegawa K, Kato T, Abe K, Hanaoka T, Miyara A, et al. Serum folate receptor alpha as a biomarker for ovarian cancer: implications for diagnosis, prognosis and predicting its local tumor expression. Int J Cancer. 2016 Apr;138(8):19942002.

26. D’Angelica M, Ammori J, Gonen M, Klimstra DS, Low PS, Murphy $L$, et al. Folate receptor- $\alpha$ expression in resectable hepatic colorectal cancer metastases: patterns and significance. Mod Pathol. 2011 Sep;24(9):1221-8.

27. Boogerd LS, Boonstra MC, Beck A), Charehbili A, Hoogstins CE, Prevoo HA, et al. Concordance of folate receptor- $\alpha$ expression between biopsy, primary tumor and metastasis in breast cancer and lung cancer patients. Oncotarget. 2016 Apr;7(14):17442-54.

28. Basal E, Eghbali-Fatourechi GZ, Kalli KR, Hartmann LC, Goodman KM, Goode EL, et al. Functional folate receptor alpha is elevated in the blood of ovarian cancer patients. PLoS One. 2009 Jul;4(7):e6292.

29. Laganà AS, Sofo V, Vitale SG, Triolo O. Epithelial ovarian cancer inherent resistance: may the pleiotropic interaction between reduced immunosurveillance and drug-resistant cells play a key role? Gynecol Oncol Rep. 2016 Sep;18:57-8.

30. Laganà AS, Colonese F, Colonese E, Sofo V, Salmeri FM, Granese R, et al. Cytogenetic analysis of epithelial ovarian cancer's stem cells: an overview on new diagnostic and therapeutic perspectives. Eur J Gynaecol Oncol. 2015;36(5):495-505. 